\title{
Qualifikation und Selektion in der Schule - Pädagogische Arbeitsorientierungen und gesellschaftliches Bewußtsein von Lehrern
}

\author{
Michael von Engelhardt \\ Soziologisches Seminar der Georg-August-Universität \\ Bürgerstraße 50 a, D-3400 Göttingen
}

$\mathrm{Z}$ u s a m m e $\mathrm{n} f$ a s s u $\mathrm{ng:}$ Aus den beiden Aufgaben der Schule - Sozialisation und Sclektion - ergeben sich zwei zentrale Bezugspunkte für die pädagogische Tätigkeit des Lehrers. In diesem Aufsatz wird auf breiter empirischer Grundlage dargelegt, wie sich die Lehrer mit diesen beiden Aufgaben auseinandersetzen. Dabei wird aufgezeigt, daß die Lehrer sehr unterschiedliche Aufgabenbestimmungen ihrer pädagogischen Tätigkeit vornehmen. Diese Unterschiede werden vor allem auf die Fachrichtung und die Schulart zurückgefuihrt. - Über die Prozesse der Sozialisation und Selektion sind die Schule und die pädagogische Tätigkeit des Lehrers mit der Gesellschaft verbunden. Deshalb werden neben pädagogischen Arbeitsorientierungen übergeordnete gesellschaftliche Deutungsmuster der Lehrer untersucht. In diesen gesellschaftlichen Deutungsmustern kommt zum Ausdruck, in welcher Weise die Lehrer die gesellschaftliche Wirklichkeit wahrnehmen und interpretieren, auf die sie die heranwachsende Generation vorbereiten. Durch die Verknüpfung der gesellschaftlichen Deutungsmuster mit der pädagogischen Aufgabenbestimmung kann die Art und Richtung des inneren Zusammenhangs zwischen den pädagogischen Arbeitsorientierungen und dem gesellschaftlichen Bewußtsein der Lehrer aufgezeigt werden.

Alle neueren Theorien zum Zusammenhang von Ausbildungssystem und Gesellschaft gehen von zwei Hauptfunktionen der Ausbildungsprozesse aus: der Funktion der Sozialisation und der Funktion der Auslese. (H. Fend, 1974) Diese beiden Funktionen bilden zwei zentrale Elemente, mit denen die Unterrichtsarbeit des Lehrers mit der Gesellschaft verbunden ist. Die konkrete Ausformung der Prozesse schulischer Sozialisation und Selektion sind unter anderem davon abhängig, in welcher Weise sich die Lehrer mit diesen beiden Funktionen auseinandersetzen. Untersuchungen zum Lehrerbewußtsein müssen deshalb darauf gerichtet sein, Sozialisationsvorstellungen herauszufinden, die die Pädagogen mit der Zielsetzung ihrer Unterrichtstätigkeit verbinden. Außerdem müssen mit solchen Untersuchungen die Formen erfaßt werden, in denen sich die Lehrer mit der Aufgabe der Selektion auseinandersetzen. Die jeweilige Interpretation dieser vorgegebenen Aufgabenstellungen, die die Lehrer vornehmen, hat objektive und subjektive Bedingungen. Sie ist von verbindlichen Vorgaben, Richtlinien, Unterrichtsstoffen und anderen Momenten der pädagogischen Arbeitssituation abhängig. In diesen Bedingungen konkretisiert sich die gesellschaftliche Organisation der Sozialisations- und Selektionsprozesse. Außerdem ist sie von subjektiven Momenten wie Lebensalter, Berufserfahrung und Ausbildung der Lehrer abhängig.

Zielsetzungen schulischer Sozialisation, mit denen die angestrebten Denk- und Handlungsweisen der auszubildenden Personen näher bestimmt werden, enthalten immer auch Vorstellungen über die Gesellschaft. Denn die angestrebten Denk- und Verhaltensweisen sind auf soziale Anwendungs- und Handlungsfelder in der Gesellschaft bezogen. Ebenso ist zu vermuten, daß ein Zusammenhang zwischen der Auseinandersetzung mit dem Ausleseprinzip und Gesellschaftsvorstellungen besteht. Daraus ergibt sich die Frage, in welchem Verhältnis die von den Lehrern vorgenommenen Interpretationen ihrer Unterrichtsaufgaben zu übergeordneten gesellschaftlichen Deutungsmustern stehen. Damit wird die Beziehung zwischen dem pädagogischen Arbeitsbewußtsein der Lehrer und ihrem gesellschaftlichen Bewußtsein thematisiert.

In diesem Aufsatz sollen empirische Ergebnisse zum pädagogischen Arbeitsbewußtsein und zum gesellschaftlichen Bewußtsein der Lehrer referiert werden, die sich auf die Funktion der Unterrichtstätigkeit und auf gesellschaftliche Deutungsmuster beziehen. Diese Ergebnisse sind im Rahmen eines Forschungsprojekts zur Arbeitsund Berufssituation von Lehrern erarbeitet worden, das über diese Themenstellung hinausgeht und am Soziologischen Seminar der Universität Göttingen durchgefürt wurde. Im Zentrum dieser Untersuchung stand die mündliche Befragung einer repräsentativen, geschichteten Stichprobe von Lehrern der allgemeinbildenden Schulen in Niedersachsen $(\mathrm{N}=1299)$. Die Verweigerungsquote lag mit $14 \%$ im Vergleich zu anderen Untersuchungen relativ niedrig. Die Interviews wurden im Sommer 1974 durchgeführt. Der dabei verwendete Fragebogen stellte eine Mischung aus 
geschlossenen und offenen Fragen dar und erforderte eine durchschnittliche Interview-Dauer von drei Stunden. Die Beteiligung der Lehrer wurde von der Mehrheit der Interviewer als interessiert bis sehr interessiert bezeichnet ${ }^{1}$.

Die nachfolgende Darstellung konzentriert sich auf die Darlegung und weitgehend immanente Interpretation der empirischen Befunde zur angefürten Fragestellung. Dabei muß auf die notwendige Einbeziehung der Ergebnisse in eine allgemeinere Theorie zum Entstehungszusammenhang von Bewußtsein ebenso verzichtet werden wie auf eine systematische Auseinandersetzung mit den vorliegenden empirischen Untersuchungen zum Lehrerbewußtsein ${ }^{2}$.

Im ersten Abschnitt werden die Qualifikationsentwürfe dargelegt, die die befragten Lehrer mit ihrer Unterrichtstätigkeit verbinden. Im zweiten Abschnitt werden zwei zentrale Momente, die die Unterrichtstätigkeit und das Bewußtsein der Lehrer stark beeinflussen - Unterrichtsfach und Schulart - in ihrer Bedeutung für die Qualifikationsziele untersucht. Im nachfolgenden Abschnitt werden die Formen behandelt, in denen sich die Befragten mit der an ihre Unterrichtstätigkeit gebundenen Auslesefunktion auseinandersetzen. Auch hier wird die Bedeutung von Unterrichtsfach und Schulart untersucht. Im vierten Abschnitt werden Deutungsmuster gesellschaftlicher Interessenauseinandersetzungen dargestellt, in denen übergeordnete gesellschaftliche Orientierungen zum Ausdruck kommen. Im fünten und abschließenden Teil wird der Zusammenhang untersucht, der zwischen den Qualifikationsentwürfen und den Interpretationen der Auslesefunktion auf der einen Seite und den gesellschaftlichen Deutungsmustern auf der anderen Seite besteht.

\section{Qualifikationsentwürfe für Arbeit und Beruf}

Ziele schulischer Sozialisation enthalten die im Ausbildungsprozeß angestrebten Kenntnisse und Fähigkeiten. Sie können als Entwürfe der Denk-

1 Thematisch gliedert sich dieses Forschungsprojekt in drei Bereiche: Unterrichtsarbeit und schulische Sozialisation; Arbeitsteilung und Kooperation im Lehrerkollegium; Interessen und gesellschaftliches Bewußtsein der Lehrer.

2 Vgl. dazu die unten angeführte Literatur und Handlungsfähigkeiten für die erwachsenen Personen verstanden werden. Werden die ausformulierten Teile von Sozialisationszielen nur auf die auszubildenden Personen begrenzt, so bleiben diese Zielsetzungen unvollständig und $a b$ strakt. Denn die auszubildenden Kenntnisse und Fähigkeiten der Heranwachsenden sind auf zukünftige Arbeits- und Lebenssituationen bezogen. Sozialisationsziele erhalten erst dann eine genauere Bestimmung, wenn die angestrebten Kenntnisse und Fähigkeiten mit der zukünftigen Arbeits- und Lebenswirklichkeit verknuipft werden. Deshalb wurden in der hier vorgestellten Untersuchung die Unterrichtsziele der Lehrer im Schnittpunkt zwischen den auszubildenden Denk- und Handlungsfähigkeiten und den gesellschaftlichen Bereichen gesucht, in denen diese Fähigkeiten Anwendung finden.

Die befragten Lehrer der allgemeinbildenden Schulen hatten die Zielsetzungen der Unterrichtstätigkeit auf die drei wichtigsten Bereiche der zukünftigen Lebenssituation ihrer Schüler zu beziehen: die Arbeits- und Berufssituation; den politisch-gesellschaftlichen Bereich; den privaten Lebensbereich. Durch diese Bezugspunkte der Sozialisationsziele sollte zum einen vermieden werden, daß sich die Beziehung zur gesellschaftlichen Anwendung im Allgemeinen verliert. Zum anderen sollte dadurch die Eingrenzung der Sozialisationsaufgabe der Schule auf die Funktion der berufsvorbereitenden Qualifikation überwunden werden. Der Vorteil dieser ausgeweiteten Aufgabenbestimmung kann allerdings innerhalb der hier veröffentlichten Arbeit noch nicht voll ausgeschöpft werden.

Im Zentrum der nachfolgenden Darstellung stehen die Sozialisationsvorstellungen der Lehrer, die sich auf die Vorbereitung für Arbeit und Beruf beziehen. Die Konzentration auf einen

Aspekt der umfassenden Sozialisationsaufgabe der Schule ist notwendig, weil nur im direkten Bezug auf ein genau bezeichnetes Anwendungsfeld die Zielsetzung der Unterrichtstätigkeit die notwendige Konkretheit erhält und weil die $\mathrm{Be}$ handlung aller Aspekte den Rahmen dieser Arbeit gesprengt hätte. Die Auswahl der auf Arbeit und Beruf gerichteten Sozialisationsziele wurde durch die besondere Bedeutung nahegelegt, die die berufsvorbereitende Qualifikation in der bildungspolitischen Diskussion und in der bildungssoziologischen Theorie der letzten Jahre erhalten hat. 
Zur Erfassung der Qualifikationsvorstellungen, die die Pädagogen mit ihrer Unterrichtstätigkeit verbinden, wurde den befragten Lehrern folgende offene Frage vorgelegt: „Was sollten die Schüler in den von Ihnen unterrichteten Fächern für die Vorbereitung auf die zukünftige Arbeits- und Berufssituation lernen? " Nach längerer Analyse der Antworten konnte herausgefunden werden, $\mathrm{da} B$ es sich bei den Aussagen der Lehrer nicht einfach nur um eine unterschiedlich ausführliche Aufzählung von Fähigkeiten und Kenntnissen handelt, die im Sinne einer Mehrfachnennung zu vercoden sind. Die komplexen Antworten der Befragten sind vielmehr als Ausdruck von jeweils ganzheitlichen Qualifikationsvorstellungen zu verstehen, die mit sehr unterschiedlichen Vorstellungen vom Verhältnis zwischen Arbeit und arbeitender Person verbunden sind. Aus der weiteren Systematisierung des empirischen Materials wurden funf verschiedene Qualifikationsvorstellungen entwickelt, denen die Aussagen der befragten Lehrer zugeordnet werden konnten. Diese verschiedenen Qualifikationsvorstellungen waren durch die Kategorie ,,kein Bezug“ zu ergänzen. Unter dieser Kategorie sind die Aussagen zusammengefaßt, in denen zum Ausdruck kommt, daß sich durch die Unterrichtstätigkeit kein spezifischer Beitrag für eine Vorbereitung der Schüler auf ihre zukünftige Arbeits- und Berufssituation leisten läßt (s. Tab. 1).

Tabelle 1: Qualifikationsziele des Unterrichts

Qualifikation für Arbeit und Beruf

kein Bezug

Arbeitstugenden und instrumentelle Qualifikationen

Instrumentelle Qualifikationen

Selbständige Anwendung der instrumentellen Qualifikationen

Selbständig-kooperative Anwendung der instrumentellen Qualifikationen

Arbeitsqualifikationen und Fähigkeit zur Durchsetzung von Arbeitnehmerinteressen

7

\begin{tabular}{lrr}
\hline Summe & 100 \\
& $\mathrm{~N}$ & 1006 \\
\hline
\end{tabular}

Ein Fünftel der befragten Lehrer an allgemeinbildenden Schulen sieht keine Möglichkeit, durch die eigene Unterrichtstätigkeit die Schüler in einer spezifischen Weise auf Arbeit und Beruf vorzubereiten. Dabei handelt es sich zum einen um eine Ablehnung dieser Sozialisationsaufgabe, die mit Hinweisen auf die niedrige Jahrgangsstufe der unterrichteten Schüler oder mit Hinweisen auf die Lerngegenstände begründet wird. Zum anderen handelt es sich um Aussagen, in denen deutlich wird, daß die Lehrer nur eine allgemeine Vermittlung von Wissen und Fertigkeiten fuir möglich halten, bei der sich kein Bezug zu Arbeit und Beruf herstellen läßt. Dieser empirische Befund macht deutlich, wie sich auf der Ebene subjektiver Zielentwürfe die Distanz der Schule von der konkreten gesellschaftlichen Realität auswirkt, auf die die heranwachsende Generation durch Bildung und Ausbildung vorbereitet werden soll.

Den positiven Vorstellungen von einer Vorbereitung der Schüler auf ihre zukünftige Arbeitsund Berufssituation ist gemeinsam, daß sie sich zunächst auf den unabdingbaren stofflichen Kern der Arbeitsfähigkeit beziehen. Dieser stoffliche Kern wird durch die instrumentelle Qualifikation gebildet, die für die Beherrschung des Arbeitsprozesses im engeren Sinne notwendig ist und die je nach Niveau und Art des Arbeitsplatzes stärker durch kognitive Fähigkeiten oder durch handwerklich-technische Fertigkeiten bestimmt ist. Bei dem Beitrag der allgemeinbildenden Schulen zur Herausbildung dieses Kerns der Arbeitsfähigkeit kann es sich immer nur um prozeßübergreifende Qualifikationen handeln, die die Basis für zunächst berufs- und dann auch prozeßspezifische Fähigkeiten und Fertigkeiten abgeben. Dieses Arbeitswissen und diese Fertigkeiten bilden die Grundlage aller von den Lehrern entwickelten Sozialisationsentwürfe.

Unterschiede zwischen den Aussagen der Lehrer ergeben sich vor allem daraus, ob und wie weitere Aspekte aufgenommen werden, die über die. sen Kern jedes Arbeitsvermögens hinausgehen. Aus diesen Unterschieden wurde die folgende Systematik der fünf Qualifikationsvorstellungen entwickelt. Im Mittelpunkt steht der rein instrumentelle Qualifikationsbegriff, der in zwei Richtungen erweitert werden kann. In der einen Richtung werden die instrumentellen Qualifikationen durch Arbeitstugenden ergänzt. In der anderen Richtung werden außerdem Aspekte der Selbständigkeit aufgefuihrt. Die damit angelegte Ausweitung wird weitergeführt durch das Auf- 
greifen einer Kooperationsfähigkeit. In einem nächsten Schritt der Ausweitung werden in die Qualifikationsvorstellungen nicht nur die bisher genannten Aspekte einer Arbeitsfähigkeit, sondern auch die Fähigkeit zur Durchsetzung von Interessen aufgenommen. Diese verschiedenen Qualifikationsvorstellungen werden im folgenden etwas eingehender dargelegt. Dabei soll durch das Zitieren von Aussagen der Befragten das Verhältnis von Kategorie und komplexer Interviewantwort aufgezeigt werden.

Der erste Qualifikationsentwurf, der als Arbeitstugenden und instrumentelle Qualifikationen bezeichnet ist, wird von mehr als einem Zehntel der Befragten dem Unterricht vorangestellt. Für die Lehrer, die sich diesen Qualifikationsbegriff zu eigen machen, ist das Verhältnis von Arbeit und arbeitender Person nicht hinreichend durch stoffliche und instrumentelle Aspekte bestimmt, so daß die Arbeitsfähigkeit durch soziale Normen, Orientierungen und Verhaltensweisen ergänzt werden muß. Die mit diesem Qualifikationsentwurf angestrebten Arbeitstugenden können sich sowohl direkt auf die Durchführung der Arbeitsaufgabe als auch auf die sozialen Beziehungen in der Arbeit richten. „Die Schüler sollen einwandfreies, selbständiges, zuverlässiges Arbeiten lernen. Selbstverantwortung für ihre Leistung, ob schlecht oder gut . . . Pflichtbewußtsein. Das Eintreten des einen für den anderen sollen sie lernen. Sie müssen sich als $\mathrm{Ge}$ meinschaft fühlen und sich darin behaupten. Sie sollen gemeinschaftskonformes Verhalten lernen." (I. 0302) Die in dieser Äußerung angesprochenen sozialen Aspekte der Arbeitstugenden werden in anderen Antworten noch deutlicher aufgegriffen. „Sie sollen ein bestimmtes Arbeitsund Pflichtverhalten lernen, bestimmte Verhaltensweisen im Umgang mit anderen: Rücksichtnahme, Respekt, Hilfsbereitschaft. Einsicht in die begrenzten Möglichkeiten, eigene persönliche Wünsche und Forderungen durchzusetzen ..." (I. 0429) „Was sollen die bei mir lernen für den Beruf? Anpassung an die gegebenen Umstände, an die Leute da, an die Vorgesetzten. Fähigkeit, von sich selbst aus eine gestellte Aufgabe zu lösen ... "(I. 0231) Die Art und Weise, wie hier Arbeitstugenden aufgegriffen und mit anderen Orientierungen verbunden werden, bewirkt die Tendenz, daß vor allem die Notwendigkeit zur Ein- und Unterordnung in vorgegebene Arbeitsaufgaben und soziale Verhältnisse in den Vorder- grund gerückt wird. Auch in den nachfolgenden Qualifikationsvorstellungen spielen Arbeitstugenden eine Rolle. Sie erhalten aber dadurch einen anderen Charakter, daß sie z.B. zu einem notwendigen Moment von Selbständigkeit oder Kooperation gemacht werden.

In der zweiten Sozialisationsvorstellung für Arbeit und Beruf wird eine Eingrenzung auf die instrumentellen Qualifikationen vorgenommen. Diese Zielsetzung der Unterrichtstätigkeit trifft auf ein Fünftel der befragten Lehrer zu. Im einzelnen geht es um unterschiedlich weit gefaßte Kulturtechniken wie Lesen und Schreiben, um sprachliches Ausdrucksvermögen, naturwissenschaftlich-technisches Wissen und um handwerkliche Fertigkeiten. ,Sie sollen lernen, sich sprachlich angemessen und fehlerfrei äußern zu können, um z.B. Lebenslauf und Bewerbungen schreiben zu können. Wenn jemand z.B. in eine Bürotätigkeit will, muß er Rechtschreibung können. Wenn einer studieren will; muß er in der Lage sein, eine wissenschaftliche Fachsprache zu erlernen und damit umgehen zu können . . . “ (I. 0024) „Handwerkliche Geschicklichkeit sollen sie erlernen: Erproben, Erkennen, statische Berechnungen, Bauen, Planen.“(I. 0122) „Die Schüler sollen durch ein gewisses naturwissenschaftliches Verständnis vorbereitet werden, um dann später technische Probleme besser bewältigen zu können. Das betrifft die Vorbereitung auf technische Berufe. Für kaufmännische Berufe sollen sie eine gewisse Fertigkeit in den Grundrechenarten und im Erkennen mathematischer Zusammenhänge erwerben." (I. 0330) In dieser zweiten Version eines Qualifikationsbegriffs wird weitgehend von den Handlungsdimensionen und den sozialen Komponenten von Arbeit abgesehen, so daß die arbeitende Person vor allem in ihrem technischen Verhältnis zum Arbeitsprozeß erfaßt wird.

Die instrumentelle Eingrenzung der Arbeitsfähigkeit wird in der dritten Qualifikationsvorstellung durch die Betonung einer Handlungsfähigkeit aufgehoben, die den Träger des Arbeitsvermögens zum selbständigen, kritischen und innovativen Umgang mit seinen Qualifikationen und seiner Arbeitssituation befähigen soll. Dieser Entwurf einer selbständigen Anwendung der instrumentellen Qualifikationen wird von knapp einem Drittel der Lehrer benutzt und gehört damit zu dem Qualifikationsbegriff, der die größ- 
te Verbreitung unter den Befragten besitzt. „,Die Schüler sollen Kritikfähigkeit erlernen, sclbständiges Arbeiten, Durchschauen der Probleme der Arbeitswelt.“ (I. 0023) ,Sie müssen lernen, neue Dinge selbständig zu erarbeiten; sie müssen verstehen, sich durchzusetzen. Gewisse Fertigkeiten müssen eben vorhanden sein, auf die sie zurückgreifen können. Sie müssen verstehen, eine Sache konzentriert zu erarbeiten. Sie müssen mit Hilfe von alten Erfahrungen neue Erfahrungen bewältigen können.“ (I. 0701) ,Sie sollen das Denken lernen und das Lernen lernen - also den Umgang mit Informationsbüchern, daß sie lernen, wie sie sich umschulen bzw. weiterbilden können." (I. 0079)

In dieser dritten Qualifikationsvorstellung kommt das unter den Lehrern verbreitete Bewußtsein zum Ausdruck, daß die bloße Vermittlung von Wissen und Fertigkeiten nicht für die Vorbereitung auf Arbeit und Beruf ausreicht. Im Unterschied zur Eingrenzung auf die instrumentellen Qualifikationen steht hier eine umfassendere Vorstellung von Arbeit und arbeitender Person im Hintergrund.

In dem nächsten Qualifikationsentwurf wird die Fähigkeit zur selbständigen und kritischen Lösung von Arbeitsaufgaben durch die Fähigkeit zu Kooperation und Kommunikation erweitert. Mit dieser Vorstellung von der selbständig-kooperativen Anwendung der instrumentellen Qualifikationen berücksichtigen die befragten Lehrer die Realität vergesellschafteter Arbeitsprozesse. In der Regel hat die arbeitende Person nicht nur mit Arbeitsgegenständen, mit Werkzeugen und Maschinen umzugehen, sondern tritt mit anderen arbeitenden Personen in eine Beziehung. Daraus leitet sich die für jede Arbeitsqualifikation notwendige Komponente einer Kooperationsund Kommunikationsfähigkeit ab, die besonders offensichtlich dort erforderlich ist, wo der Arbeitsprozeß kooperativ organisiert ist. Sie ist aber auch dort unverzichtbarer Bestandteil einer Arbeitsfähigkeit, wo Einzelarbeit vorherrscht, weil auch in diesen Fällen die individuellen Arbeitsprozesse über Anweisungen, Absprachen und Kontrollen miteinander verbunden sind.

Ein Zehntel der Befragten fuhrt diesen vierten Qualifikationsbegriff als Zielpunkt der eigenen Unterrichtstätigkeit an. ,Das Wichtigste ist selbständiges und kritisches Denken und entspre- chendes Handeln; Beherrschung der deutschen Sprache in Schrift und Wort; Zusammenarbeit im Team, Kooperation und soziales Verhalten." (I. 0522) „Ich übe mit ihnen soziales Verhalten. Die kritische Auseinandersetzung mit den Dingen, die auf sie zukommen, ist meiner Meinung nach sehr wichtig ... Sie sollen lernen, in Gruppen zu arbeiten und die Arbeit untereinander aufzuteilen - irgendetwas verantwortlich $\mathrm{zu}$ übernehmen." (I. 0994)

Die Fähigkeit zur selbständigen und kooperativen Anwendung von Arbeitswissen und instrumentellen Fertigkeiten als Zielpunkt schulischer Ausbildungsprozesse enthält gegenüber dem rein instrumentellen Qualifikationsbegriff eine doppelte Erweiterung. Zum einen wird der Träger des Arbeitsvermögens mit den Eigenschaften Selbständigkeit, Phantasie und Kritikfähigkeit ausgestattet. Zum anderen ist in diesem Qualifikationsbegriff der soziale Charakter aller Arbeitssituationen berücksichtigt. Beides - Selbständig. keit und die Fähigkeit zur Kooperation - kann zunächst nur als die Voraussetzung zur unmittelbaren Durchführung von Arbeitsprozessen verstanden werden. Die mit diesem Qualifikationsbegriff verbundenen Vorstellungen können aber auch über den unmittelbaren Arbeitsvollzug hinausgehen und sich auf die Bedingungen, Formen und auf die Herrschaftsverhältnisse von Arbeit richten. Selbständigkeit und Kritikfähigkeit kann den Arbeitsbedingungen und der Entlohnung sowie den ihnen zugrundeliegenden gesellschaftlichen Verhältnissen gelten. Die Fähigkeit zur $\mathrm{Zu}$ sammenarbeit und Verständigung mit anderen Personen kann sich zur Solidarität in der täglichen Arbeit und in den Auseinandersetzungen mit dem gemeinsamen Interessengegenüber weiter entwickeln. Diese Erweiterung wird im nächsten Qualifikationsentwurf vorgenommen.

Im fünften und letzten Entwurf einer Vorbereitung auf Arbeit und Beruf geht es um Arbeitsqualifikationen und um die Fähigkeit zur Durchsetzung von Arbeitnehmerinteressen. Mit dieser Qualifikationsvorstellung wird berücksichtigt, daß Arbeitsprozesse als eine Einheit zu verstehen sind, die eine stofflich-gegenständliche und eine gesellschaftlich-institutionelle Seite hat. Die berufsvorbereitende Sozialisation betrifft sowohl die instrumentelle Qualifikation als auch diejenigen Fähigkeiten, die zum gesellschaftlichen Verhalten in der Arbeit und gegenüber den Bedin. gungen der Arbeit notwendig sind. 
Diese umfassende Bestimmung eines Arbeitsvermögens wird von einem knappen Zehntel der Lehrer zum Bezugspunkt der Unterrichtstätigkeit gemacht. „Am wichtigsten ist, daß sie die Abhängigkeit kennen- und beurteilen lernen. Sie sollen lernen, wie man Gruppeninteressen durchsetzt. Sie sollen in der Schule zu einer angemessenen Grundausbildung kommen können, sollen bestimmte Arbeitsverfahren kennenlernen, Technologien. Wirtschaftssysteme sollen sie kennen; sie sollten die Theorie der sozialen Marktwirtschaft in der Wirklichkeit sehen können." (I. 0565) „Arbeitstechniken, Fähigkeit zur Vertretung der eigenen Interessen. Kritikfähigkeit und Fähigkeit zur Verbalisierung der Kritik. Fähigkeit, andere zu verstehen, sich selbst $z u$ bestimmen. Der Schüler soll da arbeiten können, wo er eigener Initiator ist. Soll sich mit seinen Arbeitskollegen solidarisieren und gemeinsam was mit ihnen durchsetzen können. Soll sich am Arbeitsplatz einer Hierarchie nicht kritiklos unterordnen . . ." (I. 0028) Diese Zielsetzungen, mit denen die Lehrer die aus der zukünftigen sozialen Lage ihrer Schüler resultierenden Interessen $\mathrm{zu}$ berücksichtigen suchen, schließen im Gegensatz zu den vorausgegangenen Qualifikationsentwürfen eine Vorbereitung der Lernenden auf die Wahrnehmung ihrer Rechte als Arbeitnehmer ein. Dieser Qualifikationsbegriff kann als Gegenstück zum ersten Qualifi- kationsbegriff verstanden werden, mit dem durch eine spezifische Verbindung von instrumentellen Qualifikationen mit Arbeitstugenden die Notwendigkeit einer Ein- und Unterordnung betont wird.

\section{Bedingungen der Qualifikationsentwürfe}

Die von den Lehrern entwickelten Qualifikationsentwürfe sind abhängig von den Unterrichtsfächern und der Schulart. Es ist zu vermuten, daß die Fächer in doppelter Weise die Vorstellung von einer sinnvollen Vorbereitung auf die Arbeits- und Berufsituation der Schüler bedingen. Zum einen ist mit den Stoffvorgaben des jeweiligen Faches ein spezifischer objektiver Rahmen für die Möglichkeiten einer berufsvorbereitenden Qualifizierung gegeben. Das geht sehr deutlich aus den Aussagen derjenigen Lehrer hervor, die unterschiedliche Fächer zu unterrichten haben. Zum anderen bewirken die Fächer über Ausbildung und Berufstätigkeit eine fachspezifische Sozialisation der Lehrer, die übergeordnete Wahrnehmungs- und Deutungsmuster beeinflußt. Dadurch erhalten die Zielentwürfe der pädagogischen Tätigkeit eine unterschiedliche Ausformung. Die Unterschiede zwischen den Vertretern der wichtigsten Fächergruppen sollen im folgenden dargelegt werden (s. Tab. 2).

Tabelle 2: Qualifikationsziele und Unterrichtsfach

\begin{tabular}{|c|c|c|c|c|}
\hline \multirow[t]{2}{*}{ Qualifikation für Arbeit und Beruf } & \multicolumn{4}{|c|}{ Schwerpunkt der Unterrichtsfächer } \\
\hline & Math./Nat. & Fremdspr. & Deutsch & $\begin{array}{l}\text { Gesch./ }{ }^{+)} \\
\text {Gemeinsch. } \\
\text { kunde }\end{array}$ \\
\hline kein Bezug & 21 & 26 & 12 & 19 \\
\hline $\begin{array}{l}\text { Arbeitstugenden und instrumentelle } \\
\text { Qualifikationen }\end{array}$ & 7 & 10 & 7 & 12 \\
\hline Instrumentelle Qualifikationen & 32 & 26 & 14 & 5 \\
\hline $\begin{array}{l}\text { Selbständige Anwendung der instrumentellen } \\
\text { Qualifikationen }\end{array}$ & 28 & 28 & 44 & 38 \\
\hline $\begin{array}{l}\text { Selbständig-kooperative Anwendung der } \\
\text { instrumentellen Qualifikationen }\end{array}$ & 8 & 6 & 9 & 5 \\
\hline $\begin{array}{l}\text { Arbeitsqualifikationen und Fähigkeit zur } \\
\text { Durchsetzung von Arbeitnehmerinteressen }\end{array}$ & 4 & 3 & 14 & 21 \\
\hline Summe & 100 & 100 & 100 & 100 \\
\hline 1 & 145 & 68 & 43 & 42 \\
\hline
\end{tabular}

+) unter Einschluß anderer geisteswissenschaftlicher Fächer 
Die mathematisch-naturwissenschaftlichen $\mathrm{Fä-}$ cher nehmen ein instrumentelles Verhältnis gegenüber Natur und Technik ein, das von gesellschaftlichen und historischen Kontexten und Bezügen absieht. Das fuhrt zu einer entsprechenden Ausprägung der auf die Arbeits- und Berufssituation ausgerichteten Zielsetzungen der Lehrer. So beschränkt ein Drittel der mathematischnaturwissenschaftlichen Fachlehrer die berufsvorbereitende Sozialisation auf die instrumentellen Qualifikationen, womit Arbeit vor allem als technisch-stoffliche Gegebenheit gefaßt wird. Eine fast ebenso große Gruppe erweitert die instrumentellen Qualifikationen durch die selbständige und kritische Handlungsfähigkeit. Ein Fünftel sieht keine Möglichkeit, einen spezifischen Bezug zur Arbeits- und Berufssituation der Schïler herzustellen. Die gesellschaftliche und soziale Dimension von Arbeit und Beruf wird relativ selten in die Qualifikationsziele aufgenommen, so daß nur wenige Lehrer sich auf die Kooperationsfähigkeit oder auf Aspekte der Interessenvertretung beziehen.

Die Gruppe der Fremdsprachenlehrer unterscheidet sich in den berufsvorbereitenden Sozialisationsentwürfen kaum von den mathematisch-naturwissenschaftlichen Fachlehrern. Es lassen sich die gleichen drei Schwerpunkte der Qualifikationsvorstellungen erkennen. Ein Viertel der Fremdsprachenlehrer sieht keine Möglichkeit für eine spezifische Vorbereitung auf die zukünftige Arbeitsexistenz der Lernenden. Das heißt, daß sich für die Fremdsprachenlehrer im Vergleich zu den übrigen hier untersuchten Fachlehrern die größten Schwierigkeiten bei einer inhaltlichen Berücksichtigung der Arbeits- und Berufssituation ergeben. Ein weiteres Viertel dieser Lehrer sieht in der Vermittlung instrumenteller Qualifikationen einen Beitrag zur Berufsvorbereitung, und eine etwas größere Gruppe will die Schüler zu einer Selbständigkeit in der Arbeit befähigen. Nur wenige Fremdsprachenlehrer entwickeln eine Zielsetzung, die sich auf den gesellschaftlichen Charakter von Arbeit in der Form der Kooperation und Kommunikation oder auf die in der Arbeit angelegten Interessenverhältnisse bezieht.

Von den Zielsetzungen der bisher behandelten Fachlehrer setzen sich die Qualifikationsentwürfe der Deutschlehrer deutlich ab. Das zeigt sich in der relativ geringen Verbreitung einer fehlenden Beziehung zur zukünftigen Arbeits- und Berufs- situation der Schüler. Die Unterschiede werden vor allem darin deutlich, daß die rein instrumentellen Qualifikationen eine sehr untergeordnete Rolle spielen und die erweiterte Vorstellung eines selbständigen, kreativen Umgangs mit der Arbeit den eindeutigen Schwerpunkt bildet. Ausserdem nimmt die Zielsetzung zu, die auf das Erkennen und Durchsetzen von Arbeitnehmerinteressen gerichtet ist.

In den Qualifikationsentwürfen der Deutschlehrer wird zum Teil deutlich, daß im Sprachunterricht die Fähigkeit zur Herstellung von Intersubjektivität erworben werden kann und daß eines der wichtigsten Anwendungsfelder dieser Fähigkeit im Zentrum der zukünftigen Existenz der Schüler - nämlich in der Arbeitssituation - liegen wird. Es zeigt sich aber auch die positive Funktion des Deutschunterrichts für die zu entwickelnden Fähigkeiten der Wahrnehmung und Deutung der sogenannten inneren Natur des Menschen, das heißt seiner Bedürfnisse, Interessen und Vorstellungen. Außerdem verweisen die Zielvorstellungen der Deutschlehrer darauf, daß über die Gegenstände des Sprach- und Literaturunterrichts eine Auseinandersetzung mit gesellschaftlichen Normen, Institutionen und Grundverhältnissen erfolgen kann, die in Verbindung mit den beiden anderen Elemente die Grundlage für Interessenbewußtsein, Interessenartikulation und Interessenauseinandersetzungen bilden kann.

Die Vertreter der historisch-sozialwissenschaftlichen Fächergruppe Geschichte/Gemeinschaftskunde entwickeln Zielsetzungen, die sich von den Entwürfen der Deutschlehrer vor allem dadurch unterscheiden, daß sie zum einen mit einer etwas größeren Distanz gegenüber der Arbeits- und Berufssituation und zum anderen durch ein stärkeres Aufgreifen sozialer Aspekte verbunden sind. Ein Fünftel der Lehrer dieser Fächergruppe sieht keine Möglichkeit, durch die eigene Unterrichtstätigkeit einen spezifischen Beitrag zur Berufsvorbereitung zu leisten. Dadurch haben sich die Geschichts- und Gemeinschaftskundelehrer in diesem Punkt den Zielsetzungen der Vertreter mathematisch-naturwissenschaftlicher Fächer angeglichen. Der erste gewichtige Schwerpunkt inhaltlicher Sozialisationsvorstellungen liegt bei der Fähigkeit zum selbstständigen und kritischen Umgang mit der Arbeit. Der zweite Schwerpunkt liegt bei dem fünften Qualifikationsentwurf, bei dem die Arbeitsqualifikationen durch Fähigkeiten zur Durchsetzung 
von Interessen ergänzt werden. Mit einem Fünftel findet diese Zielsetzung bei den Lehrern der historisch-sozial-wissenschaftlichen Fächer die größte Verbreitung unter den befragten Lehrern. In diesem Qualifikationsentwurf kann der spezifische Gegenstandsbezug dieser Fächergruppe, der in der Beschäftigung mit sozialen Bewegungen, mit historischen und aktuellen Normen und gesellschaftlichen Institutionen besteht, besonders deutlich zum Ausdruck kommen.

Nach der Untersuchung des Einflusses der Fächer auf die Qualifikationsvorstellungen der Lehrer soll im folgenden die Bedeutung der Schularten aufgewiesen werden. Die Vorstellungen der Lehrer an der Grundschule setzen sich in zwei Momenten von den Zielsetzungen der übrigen Lehrer ab, die sich beide aus der größeren Distanz gegenüber der zukünftigen Arbeits- und Berufssituation der Schüler erklären lassen. Die Lehrer, deren Unterrichtsschwerpunkt in den ersten beiden Jahrgangsstufen liegt, sehen relativ häufig keine Möglichkeit einer spezifischen berufsvorbereitenden Sozialisation (kein Bezug: 27\%). Der fehlende Bezug geht aber deutlich bei den Leh-

Tabelle 3: Qualifikat ionsziele und Schulart

\begin{tabular}{llll}
\hline Qualifikation für & \multicolumn{4}{l}{ Lehrer der oberen Jahrgangsstufen } \\
Arbeit und Beruf & $\begin{array}{lll}\text { Haupt- } \\
\text { schule }\end{array}$ & $\begin{array}{l}\text { Real- } \\
\text { schule }\end{array}$ & Gymnasium \\
& $(\mathrm{Jg} .8-9)$ & $(\mathrm{Jg} .8-10)$ & $(\mathrm{Jg} .11-13)$ \\
\hline
\end{tabular}

kein Bezug

13

23

28

Arbeitstugenden und instrumentelle Qualifikation

Instrumentelle Qualifikationen

Selbständige Anwendung der instrumentellen Qualifikationen

Selbständig-kooperative Anwendung der instrumentellen Qualifikationen

Arbeitsqualifikationen und Fähigkeit zur Durchsetzung von Arbeitnehmerinteressen

\begin{tabular}{llllr}
\hline Summe & $\%$ & 100 & 100 & 100 \\
& $\mathrm{~N}$ & 113 & 110 & 36 \\
\hline
\end{tabular}

rern zurïck, deren Unterrichtsschwerpunkt in den Jahrgangsstufen drei und vier liegt. Außerdem greifen die Lehrer der Grundschule seltener als ihre Kollegen an der Sekundarstufe den um die Dimension der Interessendurchsetzung angereicherten Qualifikationsbegriff auf (3\%).

Die Lehrer der drei weiterführenden Schulen Hauptschule, Realschule und Gymnasium entwickeln unterschiedliche Vorstellungen einer benufsvorbereitenden Sozialisation (s. Tab. 3). Diese Differenzen bilden sich mit wachsender Nähe zum Schulabschluß prägnanter heraus. Das bedeutet, daß sich die Lehrer, deren Unterrichtsschwerpunkt in den jeweiligen Abschlußklassen der drei weiterführenden Schularten liegt, am deutlichsten in ihren Zielsetzungen unterscheiden. Die Unterschiede gehen auf drei Tendenzen zurück. $\mathrm{Zu}$ nächst einmal nimmt der fehlende Bezug auf die Arbeits- und Berufssituation im Übergang von der Hauptschule zur Realschule und im Übergang von der Realschule zum Gymnasium zu. In einer zweiten Tendenz nehmen in gegenläufiger Richtung Sozialisationsvorstellungen $a b$, in denen die Arbeitstugenden eine besondere Beto. nung erhalten. Und schließlich sinkt die Berücksichtigung von Problemen der Interessenvertretung von einem Zehntel bei den Lehrern der Abschlußklassen an der Hauptschule und an der Realschule auf drei Prozent bei den Lehrern an der gymnasialen Oberstufe. Diese empirischen Befunde deuten darauf hin, daß sich in den subjektiven Zielentwürfen der Lehrer die Unterschiede auswirken, die sich aus der schulartspezifischen Beziehung zur gesamtgesellschaftlichen Realität ergeben. Da an die einzelne Schulart des dreigliedrigen Schulsystems nur ganz bestimmte Berufsperspektiven geknüpft werden können, wird auch nur ein bestimmter Ausschnitt aus der gesellschaftlichen Hierarchie von Arbeits- und Lebenssituationen zum Bezugspunkt der Qualifikationen.

Für die Hauptschullehrer ist die Beziehung schulischer Lehr- und Lernprozesse zur zukünftigen Arbeits- und Berufssituation am engsten gegeben, so daß sie relativ selten keine Möglichkeit für eine berufsvorbereitende Sozialisation sehen. Außerdem unterscheiden sie sich von ihren Kollegen an den beiden anderen Schularten darin, daß sie häufiger die Verknüpfung von instrumentellen Qualifikationen mit Arbeitstugenden aufgreifen. Damit betonen sie stärker als die ande- 
ren Pädagogen die Notwendigkeit einer Integration in vorgegebene Aufgaben und soziale Beziehungen. Auf der anderen Seite greift ein Zehntel die Zukunftsperspektive der Schüler in dem Sinne auf, daß im Unterricht auf Probleme der Interessenvertretung vorbereitet werden soll. Darin unterscheiden sich die Hauptschullehrer von den Gymnasiallehrern.

\section{Im Übergang von der Hauptschule zur Real-} schule verschiebt sich die objektive Qualifikationsfunktion in die Richtung höherer Stufen der gesellschaftlichen Hierarchie von Arbeits- und Berufssituationen. In den Zielsetzungen der Lehrer zeigt sich eine stärkere Distanz gegenüber der Arbeits- und Berufssituation und eine abnehmende Bedeutung von Arbeitstugenden. Im Übergang von der Realschule zum Gymnasium wird diese Entwicklungslinie weiter fortgesetzt. Sie wird dadurch ergänzt, daß die Vorbereitung auf die Durchsetzung von Arbeitnehmerinteressen nahezu keine Rolle mehr in den Zielsetzungen der Gymnasiallehrer spielt.

\section{Auseinandersetzung mit der Auslesefunktion}

Mit der im Schulsystem vorgenommenen Auslese unter den Schülern wird die Verteilung der heranwachsenden Generation auf die gesellschaftliche Hierarchie von Arbeits- und Lebenssituationen vorbereitet. An diesem Prozeß nimmt jeder einzelne Lehrer durch die Bewertung der Lernleistungen seiner Schüler teil. Aus der Summe dieser Beurteilungen ergibt sich die Schullaufbahn des Schülers, in der ihm Chancen auf weitere Bildungsgänge eingeräumt oder verweigert werden. Die Funktion der Selektion ist ebenso wie die der Qualifikation Bestandteil der pädagogischen Tätigkeit (M.v. Engelhardt, 1978) und verbindet diese mit der außerhalb des pädagogischen Feldes liegenden Gesellschaft.

Sind den Lehrern schon bei der Gestaltung der Qualifikationsentwürfe relativ enge Grenzen durch die Inhalte und Arbeitsbedingungen ihrer Tätigkeit gesetzt, so werden die Grenzen noch enger gezogen, wenn es um die Aufgabe der Selektion geht. Zum einen sind die Kriterien, nach denen die Auslese erfolgt, dem Einfluß einzelner Pädagogen weitgehend entzogen. Zum anderen sind die Möglichkeiten, die der Lehrer in seiner Arbeit hat, um durch Förderungsmaßnahmen die Voraussetzungen der Auslese zu verbessern und ihre Folgen abzuschwächen, sehr eingeschränkt. Deshalb kann sich für den einzelnen Lehrer aus der Forderung nach einer umfassenden Förderung aller Schüler auf der einen Seite und aus der Notwendigkeit einer Selektion unter den Lernenden auf der anderen Seite cin Dilemma entwickeln, das zu einer Belastung der Unterrichtstätigkeit führt. Dieses Dilemma wurde in dieser Untersuchung zum Ausgangspunkt genommen, um die Auseinandersetzung der Befragten mit der Selektionsfunktion zu erfassen. Dabei wurde auf die Aussage eines Lehrers zurückgegriffen, die wir im Rahmen von explorativen Vorgesprächen aufgenommen hatten.

Den Befragten wurde folgende Frage vorgelegt: „Ein Lehrer hat in einem Gespräch mit uns gesagt: ,Für mich besteht ein unauflösliches Dilemma darin, daß ich einerseits alle Schüler fördern soll und daß ich andererseits gezwungen bin, über die Leistungsbewertung eine Auslese unter den Schülern zu treffen. 'Trifft diese Aussage auch auf Sie zu, oder würden Sie das anders sehen?" Während sich die Hälfte der Lehrer mit diesem Dilemma in der pädagogischen Arbeit konfrontiert sieht, besteht für eine fast ebenso große Gruppe kein konfliktreiches Verhältnis zwischen Förderung und Auslese. Nur sehr wenige Lehrer lehnen die Problemstellung mit dem Hinweis $a b$, daß mit ihrem Unterricht keine Auslese verbunden ist (s. Tab. 4).

Tabelle 4: Verarbeitung der Selektionsfunktion

\begin{tabular}{lc}
\hline $\begin{array}{l}\text { Dilemma zwischen } \\
\text { Förderung und Auslese }\end{array}$ & $\begin{array}{l}\text { Lehrer der allgemein- } \\
\text { bildenden Schulen }\end{array}$ \\
\hline $\begin{array}{l}\text { Besteht } \\
\text { Besteht nicht, weil kein }\end{array}$ & 49 \\
$\begin{array}{l}\text { Widerspruch } \\
\text { Besteht nicht, weil keine }\end{array}$ & 44 \\
Auslese & 5 \\
Weiß nicht & 2 \\
\hline Summe \% & 100 \\
\hline
\end{tabular}

Die erste Gruppe der Lehrer macht im Unterricht die Erfahrung, daß den Schülern zur Entwicklung der in ihnen angelegten Möglichkeiten und zum Ausgleich unterschiedlicher Lernvoraussetzungen mehr und andere Lernchancen geboten werden müßten, als das gegenwärtig möglich ist. Sie tritt in ein problematisierendes 
Verhältnis zu den Formen und Kriterien der Leistungsbewertung. Die Lösungsperspektiven der Lehrer, die aus den Antworten auf eine entsprechende Nachfrage hervorgehen, beziehen sich sowohl auf die Formen und Kriterien der Leistungsbewertung als auch auf die Formen der Förderung. Dabei wird zunächst der individuelle Handlungsspielraum soweit wie möglich genutzt. Je schärfer aber die vorherrschende Praxis der Selektion problematisiert wird, desto deutlicher werden die Schranken eines individuellen Ausgleichs erlebt. Damit ist die Gefahr verbunden, daß der erlebte Konflikt resignativ als unabänderliche Tatsache interpretiert wird. Diese Gefahr kann nur durch eine überindividuelle Veränderungsperspektive eingeschränkt werden. $D a ß$ diese Lehrer ein verstärktes Interesse an Schulreformen zeigen, die auf eine Veränderung der Mechanismen und Bedingungen der Auslese abzielen, verweist auf das Vorhandensein einer solchen Perspektive.

Die andere Gruppe der Lehrer, die ebenfalls fast die Hälfte der Befragten umfaßt, sieht vor allem deshalb keinen Widerspruch zwischen der Aufgabe der Förderung und der Selektion, weil sich für sie die Auslese nach den Begabungen und den erbrachten Leistungen richtet und weil dadurch zu Recht immer wieder aus den Lernenden diejenigen ausgewählt werden, für die eine weiterführende Förderung ergiebig erscheint. Damit wird eine Ausprägung des Leistungsprinzips vertreten, nach dem sich der Schüler das Recht auf die Teilnahme an Ausbildungsprozessen durch erfolgreiches Lernen erwerben muß. Indem mit diesem Prinzip allen Schülern die gleichen Lernbedingungen in der Schule angeboten werden und die Möglichkeit ihrer längerfristigen Nutzung vom Lernerfolg abhängig gemacht wird, findet ein Gleichheitsgrundsatz Anwendung, der durch das Absehen von den unterschiedlichen Lernvoraussetzungen die Reproduktion sozialer Ungleichheit im Bildungssystem verschleiert. Die Lehrer, die die Auslesefunktion auf diese Weise interpretieren, sind weniger als die anderen Lehrer an Reformen interessiert, die auf eine Veränderung der Auslesemechanismen gerichtet sind. Sie bestehen vielmehr auf eine Beibehaltung oder gar Verschärfung der tradierten Formen.

Ebenso wie bei den Qualifikationsentwürfen zeigt sich auch bei der Beurteilung der Auslesefunktion eine Abhängigkeit der Lehreraussagen vom Unterrichtsfach und von der Schulart.

Beide Momente führen zu einer unterschiedlichen Auswirkung der Selektion auf den Unterricht und zu einer unterschiedlichen Wahrnehmung durch die Lehrer (s. Tab. 5).

Tabelle 5: Verarbeitung der Selektionsfunktion und Unterrichtsfach

Dilemma zwischen

Förderung und Auslese
Math./Nat.
Schwerpunkt der Unterrichtsfächer

Fremdspr.
Gesch./ ${ }^{+}$ Gemeinsch kunde

\begin{tabular}{|c|c|c|c|c|c|}
\hline \multirow{2}{*}{\multicolumn{2}{|c|}{$\begin{array}{l}\text { Besteht } \\
\text { Besteht nicht, weil kein Widerspruch }\end{array}$}} & 32 & 35 & 48 & 51 \\
\hline & & 59 & 59 & 50 & 44 \\
\hline \multicolumn{2}{|c|}{ Besteht nicht, weil keine Auslese } & 4 & 4 & 2 & 2 \\
\hline \multicolumn{2}{|c|}{ Weiß nicht } & 4 & 1 & 0 & 2 \\
\hline \multirow[t]{2}{*}{ Summe } & $\%$ & 100 & 100 & 100 & 100 \\
\hline & $\mathbf{N}$ & 145 & 68 & 42 & 43 \\
\hline
\end{tabular}

+) unter Einschluß anderer geisteswissenschaftlicher Fächer

Unter den hier untersuchten Fachlehrern zeigt sich eine deutliche Zweiteilung. Für die Lehrer der Fremdsprachen und der mathematisch-naturwissenschaftlichen Fächer ist das Dilemma seltener gegeben als für die Lehrer der Fächer
Deutsch, Geschichte/Gemeinschaftskunde. Die Definition des Lernens und der Lernergebnisse, die mit den Fremdsprachen und den mathematisch-naturwissenschaftlichen Fächern verbunden ist, scheint zu einer abgeschwächten Wahrneh- 
mung der Diskrepanz zwischen den zu fördernden Fähigkeiten und Fertigkeiten und den erbrachten Leistungen zu führen, an denen die Auslese ausgerichtet wird. Es ist zu vermuten, daß dies auf zwei Bedingungen zurückgeht. Zum einen sind in diesen Fächern die Probleme der angemessenen Übersetzung von qualitativen Lernleistungen in die Formen der abstrahierenden Leistungsmessung und Leistungsbeurteilung nicht so stark ausgeprägt wie in den anderen Fächern. Zum anderen werden die Unterschiede in den psycho-sozialen Lernvoraussetzungen der Schüler, die im außerschulischen Herkunftsmilieu liegen, weniger stark erfahren.
In den Fächern Geschichte, Gemeinschaftskunde und Deutsch sind die Lehrer im Vergleich zur ersten Fächergruppe wesentlich stärker mit den Schwierigkeiten konfrontiert, das Ergebnis des Lernens auf eine angemessen Weise in Leistungsmessung und Leistungsbeurteilung zu überführen. Gleichzeitig werden mehr Ausschnitte der Person des Schülers und seiner bisherigen psychosozialen Entwicklungsgeschichte in die offenen Teile des Unterrichts einbezogen. Die durch diese Fächer bedingte Unterrichtswirklichkeit und Wahrnehmungsstruktur führt zu einem verstärkten Bewußtsein vom pädagogischen Dilemma zwischen Förderung und Auslese.

Tabelle 6: Verarbeitung der Selektionsfunktion und Schulart

\begin{tabular}{|c|c|c|c|c|c|}
\hline \multicolumn{2}{|c|}{ Dilemma zwischen Förderung und Auslese } & $\begin{array}{l}\text { Grund- } \\
\text { schule }\end{array}$ & $\begin{array}{l}\text { Haupt- } \\
\text { schule }\end{array}$ & $\begin{array}{l}\text { Real- } \\
\text { schule }\end{array}$ & Gymnasium \\
\hline \multicolumn{2}{|l|}{ Besteht } & 58 & 52 & 46 & 34 \\
\hline \multicolumn{2}{|c|}{ Besteht nicht, weil kein Widerspruch } & 36 & 42 & 50 & 56 \\
\hline \multicolumn{2}{|c|}{ Besteht nicht, weil keine Auslese } & 4 & 4 & 4 & 5 \\
\hline \multicolumn{2}{|c|}{ Weiß nicht } & 2 & 2 & 0 & 4 \\
\hline \multirow[t]{2}{*}{ Summe } & $\%$ & 100 & 100 & 100 & 100 \\
\hline & $\mathbf{N}$ & 241 & 244 & 246 & 223 \\
\hline
\end{tabular}

Von allen Lehrern der allgemeinbildenden Schulen fühlen sich die Grundschullehrer am stärksten von dem Dilemma in ihrer Arbeit belastet (s. Tab. 6). Durch das Alter und die soziale Heterogenität der Schüler ist diesen Lehrern die Notwendigkeit einer breiten Förderung besonders stark gegenwärtig, der sie wegen der unzulänglichen organisatorischen, inhaltlichen und personellen Bedingungen ihrer Arbeit nicht hinreichend nachkommen können. Außerdem ist ihnen die anstehende Verteilung ihrer Schüler auf die drei weiterführenden Schularten klar im Bewußtsein, die für den weiteren Ausbildungsweg und für die zukünftigen Arbeits- und Lebenssituationen von entscheidender Bedeutung ist. Die Lehrer der drei weiterführenden Schularten setzen sich in unterschiedlicher Weise mit der Auslesefunktion auseinander. Mit aufsteigender Hierarchiestufe im Schulsystem nimmt das problematische Verhältnis ab, das die Lehrer zum Dilemma zwischen Förderung und Auslese eingehen.

Die Hauptschullehrer fühlen sich am stärksten durch dieses Dilemma in ihrer Arbeit belastet. $D_{a}$ sie es mit denjenigen Schülern zu tun haben, auf die sich die negativen Folgen der Auslese konzentrieren, tritt ihnen am ehesten der Widerspruch zwischen den Möglichkeiten und Notwendigkeiten einer Ausbildung entgegen. Sie erfahren in ihrer pädagogischen Tätigkeit sehr unmittelbar, wie wenig die praktizierten Formen und Kriterien der Selektion geeignet sind, ihren Schülern das Recht auf gleiche Bildungsmöglichkeiten zu sichern. Diese Erfahrung geht bei den Realschullehrern und den Gymnasiallehrern immer weiter zuriick, so daß sich der Widerspruch immer stärker aus der Arbeitserfahrung verliert. Für die Verarbeitung der Auslesefunktion könnte aber noch ein anderer Aspekt von Bedeutung sein.

Es ist zu vermuten, daß die Bereitschaft des Pädagogen zur Problematisierung der Selektionsfunktion in dem Maße abnimmt, wie sich die schulische Auslese positiv auf die Unterrichtstätigkeit auswirkt. Die schulische Selektion bedeutet eine immer wieder neu vorgenommen Aussonderung derjenigen Schüler, die sich in die jeweiligen Formen und Inhalte des Unterrichtens nicht integrieren lassen. Auf diese Weise entsteht in den oberen Bereichen des Schulsystems das beste Verhältnis zwischen den Fähigkeiten und Verhaltensweisen der Lernenden und den 
schulischen Anforderungen. Da nun gerade von diesem Verhältnis das Ausmaß von Belastungen im Unterricht abhängt, kann das Urteil des Pädagogen zur Selektionsfunktion durch sein unmittelbares Arbeitsinteresse bestimmt werden. Das führt mit aufsteigender Stufe im hierarchisch gegliederten Schulsystem zu einer positiven Anbindung an die gesellschaftliche Auslesefunktion der Schule.

\section{Deutungsmuster gesellschaftlicher Inter- essenunterschiede}

In den vorausgegangenen Abschnitten sind die Qualifikationsentwürfe und Formen der Auseinandersetzung mit der Auslesefunktion untersucht worden. Dabei handelt es sich um zwei zentrale Aufgaben der Unterrichtstätigkeit, über die diese ihren gesellschaftlichen Charakter erhält. Die dargelegten Vorstellungen und Interpretationen der befragten Lehrer sind zentrale Momente des pädagogischen Arbeitsbewußtseins, die eine orientierende Funktion für die Unterrichtspraxis besitzen (vgl. z.B. v. Engelhardt, 1979). Außerdem sind in den untersuchten pädagogischen Zielsetzungen und Auseinandersetzungsformen wichtige Momente eines übergeordneten gesellschaftlichen Bewußtseins enthalten, so daß sie a's die Vermittlungsstelle $z$ wischen pädagogischem Arbeitsbewußtsein und gesellschaftlichen Orientierungen verstanden werden können.

In diesem Abschnitt soll nun das über die pädagogische Tätigkeit hinausgehende gesellschaftliche Bewußtsein der Lehrer behandelt werden, um daran anschließend den Zusammenhang zwischen gesellschaftlichen Deutungsmustern und Interpretationen der pädagogischen Aufgaben untersuchen zu können.

Das Erfassen übergeordneter gesamtgesellschaftlicher Deutungsmuster muß sich auf einen zentralen Aspekt gesellschaftlicher Wirklichkeit beziehen. In der hier vorgestellten Untersuchung wurde davon ausgegangen, daß gesellschaftliche Deutungsmuster relativ gut zum Ausdruck kommen, wenn sich die Befragten zur Tatsache gesellschaftlicher Interessenauseinandersetzungen äußern. Den befragten Lehrern wurden deshalb die folgenden beiden Aussagen zur Beurteilung vorgelegt ${ }^{3}$. Die erste Aussage lautete: ,In unserer gegenwärtigen Gesellschaft gibt es die verschiedensten Interessengruppen, die sich teilweise auch bekämpfen. Charakteristisch für unsere Gesellschaft ist jedoch, daß sich die Interessen in Form von Kompromissen alle einigermaßen zufriedenstellend ausgleichen lassen!" Dagegen wurde eine zweite Aussage gesetzt: „Natürlich gibt es in unserer gegenwärtigen Gesellschaft viele Interessenunterschiede, die sich durch Kompromisse regeln lassen. Charakteristisch für unsere Gesellschaft sind jedoch die grundlegenden Interessengegensätze zwischen gesellschaftlichen Gruppen, die sich nicht durch Kompromisse ausgleichen lassen!" Die interviewten Lehrer hatten sich zunächst einer dieser beiden Aussagen zuzuordnen. Außerdem gab es die Möglichkeit, die vorgegebene Alternative abzulehnen. In einem zweiten Schritt sollten die Lehrer ausführliche Begründungen geben und in einem dritten Schritt wurde nach Lösungsperspektiven gefragt.

Aus der Analyse des empirischen Materials wurde deutlich, daß sich nur dann ganzheitliche Deutungsmuster erkennen lassen, wenn die formale Zuordnung zur vorgegebenen Alternative und die Aussagen zur ersten und zur zweiten Nachfrage gleichzeitig betrachtet und dabei als eine einheitliche Darlegung des Problems verstanden werden. Bei der Bildung der Auswertungskategorien ging es im wesentlichen um drei Dimensionen: Ursachen von Interessenunterschieden und Art der Interessen; Art der thematisierten Interessengruppen; Möglichkeit der Vermittlung bzw. des Abbaus von Interessenunterschieden. Daraus wurden vier Deutungsmuster gesellschaftlicher Interessenunterschiede entwickelt, denen nahezu alle Aussagen der Befragten zugeordnet werden konnten (s. Tab. 7). Die genauere Charakterisierung dieser Deutungsmuster wird im folgenden durch ausführliche Interview-Zitate konkretisiert und belegt. Dabei ist zu berücksichtigen, daß es sich um Antworten auf zwei nacheinander gestellte Fragen handelt. Die Nachfrage, die auf Vermittlungs- und Lösungsperspektiven zielte, ist durch ,,(N:)“ gekennzeichnet.

3 Der hier verwendete Zugang zum gesellschaftlichen Bewußtsein wurde aus einer anderen Untersuchung übernommen; vgl. M.v. Engelhardt und R.-W. Hoff.mann 1974: $428 \mathrm{ff}$. 
Tabelle 7: Gesellschaftliche Deutungsmuster

\begin{tabular}{ll}
\hline $\begin{array}{l}\text { Deutungsmuster gesellschaftlicher } \\
\text { Interessenunterschiede }\end{array}$ & $\begin{array}{l}\text { Lehrer der all- } \\
\text { gemeinbildenden } \\
\text { Schulen }\end{array}$ \\
\hline
\end{tabular}

Individueller Antagonismus

Individueller Pluralismus

Sozio-ökonomische Sozialpartnerschaft

Sozio-ökonomische Ungleichheit

Sonstiges

4

\begin{tabular}{lrr}
\hline Summe & $\%$ & 100 \\
& $\mathrm{~N}$ & 1006 \\
\hline
\end{tabular}

Die Deutungsmuster der befragten Lehrer lassen sich zunächst nach einem wichtigen Kriterium in zwei Gruppen unterteilen. Auf der einen Seite stehen Deutungsmuster, mit denen Interessenauseinandersetzungen im wesentlichen auf Individuen, ihr Streben nach Macht und Reichtum sowie ihre Weltanschauungen zurückgeführt werden. Auf der anderen Seite stehen Deutungsmuster, mit denen soziale Gruppen und sozioökonomische Grundlagen aufgegriffen werden. Mit der ersten Vorstellung ist die Tendenz verbunden, daß sich die Gesellschaft in eine Summe von Individuen auflöst, die sich in der Durchsetzung ihrer Interessen mehr oder minder behindern. Wenn Gruppen angeführt werden, so geschieht das relativ allgemein und unbestimmt. Soziale und ökonomische Dimensionen, durch die die einzelnen Gesellschaftsmitglieder zu sozialen Gruppen, Klassen und Schichten zusammengefaßt und auch voneinander getrennt werden, bleiben weitgehend ausgeklammert. Daraus entsteht die Gefahr, daß die Bestimmung der Gesellschaft einen ahistorischen und unspezifischen Charakter erhält und daß gesellschaftliche Auseinandersetzungen nur mit allgemeinen Wesensmerkmalen des Menschen begründet werden. In diese erste Gruppe der Deutungsmuster fallen die Aussagen von drei Fünfteln der befragten Lehrer. Innerhalb dieser Gruppe muß aber noch eine wichtige Unterscheidung getroffen werden, die sich nach der Lösungsperspektive richtet, mit der die aus den Interessenunterschieden resultierenden gesellschaftlichen Konflikte versehen werden. Dabei zeigt sich ein Zusammenhang zwischen der Lösungsperspektive und den Ursachen, die für die Interessenauseinandersetzungen angegeben werden.
Mit dem ersten Deutungsmuster, das als individueller Antagonismus bezeichnet wird und auf ein knappes Fünftel der Befragten zutrifft, werden die Interessen der Menschen so interpretiert, daß zwischen ihnen kaum Vermittlungsprozesse denkbar sind. Das im Menschen angelegte ökonomische Interesse, sein Streben nach Macht und die ihn leitenden Ideologien stehen in einem antagonistischen Verhältnis zu den Interessen der anderen Gesellschaftsmitglieder. Gleichzeitig wird der Interessenkampf als eine Naturkonstante gefaßt. ,Der Interessengegensatz liegt im Menschen selbst, in der genetischen Entwicklung: angeborene Interessenmotivation, angeborene unterschiedliche Begabung, angeborene unterschiedliche Intelligenz. (N:) Eine Beseitigung der Interessengegensätze ist nicht möglich. Das liegt an den angeborenen Eigenschaften des Menschen, die sich nicht beseitigen lassen." (I. 1147) ,Gibt es überhaupt eine Gesellschaftsform ohne Bekämpfung? So banal es sich anhört es ist fast eine Naturkonstante. Selbst in Gesellschaftsformen, wo von oben angestrebt wird, sich nicht zu bekämpfen, entstehen immer wieder Interessenkämpfe. Ich sehe es fast in der Schöpfung des Menschen verankert. Es hat immer Kampf gegeben ...“(1. 0860) „, Die Ursache von Interessengegensätzen: Das ganz persönliche Streben nach Macht, Einfluß und hohem Lebensstandard. (N:) Die Beseitigung ist nicht möglich, sonst müßte man den Menschen ändern."

(I. 1154 )

Da bei diesem Deutungsmuster eine spezifische historische und soziale Begründung der Interessenunterschiede fehlt, können sich auch in der Regel keine Vermittlungs- und Veränderungsperspektiven abzeichnen. Das kann dann auch dazu führen, daß den gesellschaftlichen Institutionen und Normen nicht so sehr die Funktion zugewiesen wird, Verständigungs- und Auseinandersetzungsprozesse zu ermöglichen und eine Veränderung sozialer Wirklichkeit einzuleiten. Sie erhalten vielmehr die Funktion der bloßen Konfliktvermeidung.

Mit dem zweiten Deutungsmuster, das von zwei Fünfteln der Befragten vertreten wird, werden die Interessen und Ideologien der Gesellschaftsmitglieder so gefaßt, daß zwischen ihnen Vermittlungsprozesse und Kompromisse möglich sind. Dabei wird aber nicht auf konkret zu bezeichnende soziale Gruppen oder gesellschaft- 
liche Bezuige eingegangen. Dieses Deutungsmuster wird deshalb als individueller Pluralismus bezeichnet. „Nicht alle haben die gleichen Interessen. Es gibt Auseinandersetzungen, weil die Menschen nicht gleich sind . . ( $(\mathrm{N}:)$ Kompromisse sind möglich, weil es Verhandlungen gibt - aus politischer Notwendigkeit. Wenn man miteinander leben will, muß man miteinander verhandeln - aus politischer Vernunft. Wer keine Diktatur will, muß verhandeln." (I. 1067) „Jeder denkt hauptsächlich an seinen Vorteil und meint, seine Ansicht sei richtig. (N:) Kompromisse sind durch Vernunft und Einsicht der Partner möglich.“ (I. 0878) „Weltanschauliche Gegensätze, mangelnde Toleranz und Unfähigkeit, sachlich miteinander $z u$ sprechen, sind die Ursachen von Auseinandersetzungen (N:). Kompromisse ergeben sich aus der Notwendigkeit, eine Entscheidung zu fällen ... Durch Verhandlungen und zeitweiliges Zurückstellen eigener Interessen, ohne die Zielsetzung aus dem Auge zu verlieren ..." (I. 1188) Im Vergleich zum ersten Deutungsmuster werden im zweiten Deutungsmuster die Ursachen von Interessenkonflikten etwas stärker mit einem sozialen Charakter versehen. Vor allem aber werden die Vertreter von Interessen mit einer ,Gesellschaftlichkeit" ausgestattet, die sie in die Lage versetzt, sich auf der Grundlage einer Gemeinsamkeit und über Toleranz und soziale Orientierungen miteinander zu verständigen. Deshalb machen es die Einsicht in gemeinsame Interessen und die Orientierung an übergeordneten Zielen möglich, daß sich die Interessenunterschiede in der Regel in Kompromisse überführen lassen.

Den beiden ersten Deutungsmustern gesellschaftlicher Interessenauseinandersetzungen stehen die beiden nachfolgenden Erklärungsversuche gegenüber, mit denen soziale Gruppen und sozioökonomische Grundlagen explizit aufgegriffen werden. Damit erhält das interessengeleitete Individuum einen spezifischen sozialen Charakter und eine soziale Einbettung. Gleichzeitig wird Gesellschaft als eine übergeordnete Einheit erfaßt. Indem von den Lehrern mit diesen beiden Deutungsmustern vor allem das Verhältnis der beiden gesellschaftlichen Hauptgruppen - Arbeitnehmer und Vertreter des Kapitals - in den Mittelpunkt gerückt wird, wird die historisch-soziale Besonderheit dieser Gesellschaft bestimmt.

Mit dem dritten Deutungsmuster, das hier als sozio-ökonomische Partnerschaft bezeichnet wird und das auf ein Fünftel der befragten Lehrer an allgemeinbildenden Schulen zutrifft, werden die gesellschaftlichen Auseinandersetzungen mit dem Kampf der sozialen Gruppen um ökonomische und soziale Vorteile begründet. Die daraus resultierenden Interessenunterschiede lassen sich aber in der Regel in Kompromisse überführen, weil die ökonomische Situation und die damit verbundene Gemeinsamkeit der Interessen es erzwingen und weil eine entsprechende sozialpartnerschaftliche Orientierung dieses möglich macht. ,Es gibt Interessenauseinandersetzungen, weil die Gruppen verschiedene Ziele haben, weil wir in dem System der freien Marktwirtschaft leben. Da gibt es eben Unternehmer auf der einen und Lohnabhängige auf der anderen Seite. (N:) Kompromisse sind möglich, weil das gesamtstaatliche Interesse Priorität hat." (I. 0844) „Interessenkämpfe ergeben sich aus Mangel an Einsicht in die sozialen Gegebenheiten. Arbeitnehmer und Arbeitgeber sollten sich z.B. nicht als Freiwild ansehen. (N:) Der Kompromiß ist möglich durch die Notwendigkeit zur Zusammenarbeit. Keine Gruppe kann einen Alleingang gehen. Jede Gruppe, die Vorteile für sich herausholt, muß bedenken, daß es zu Lasten einer anderen Gruppe geht.“ (I. 0880) ,Es liegt an der Verteilung des Kapitals und der Produktionsmittel. Nur dadurch ergeben sich die Unterschiede. (N:) Kompromisse ergeben sich, weil wir aufeinander angewiesen sind. Es besteht die Abhängigkeit beider Gruppen voneinander. Jeder braucht den anderen, deshalb müssen sich beide arrangieren." (I. 1107)

Mit dem dritten Deutungsmuster der sozio-ökonomischen Partnerschaft ist im Prinzip die Annahme einer Gleichrangigkeit der im Interessenkampf liegenden Gruppen verbunden. Eine soziale Ungleichheit wird zumindest nicht hervorgehoben. Demgegenüber bildet die sozio-ökonomische Ungleichheit den Ausgangspunkt des vierten Deutungsmusters, das von einem knappen Fünftel der befragten Lehrer verwendet wird.

Mit diesem Erklärungsversuch werden gesellschaftliche Auseinandersetzungen auf soziale Ungleichheit zurückgefuihrt, die es je nach objektiver Interessenlage abzubauen oder aufrechtzuerhalten gilt. Aufgabe der Politik ist es, diese soziale Unglsichheit in einer kurz- und längerfristigen Perspektive einzuschränken. ,Wir leben in einer Gesellschaft, wo das Privateigentum eine 
Grundkategorie ist und deshalb Machtunterschiede zwischen Lohnarbeit und Kapital entstehen, die unaufhebbar sind. Kompromisse sind nur soweit möglich, wie sie die Machtstrukturen nicht anruhren. Wir haben keine pluralistische Gesellschaft in dem Sinne, daß alle die gleichen Möglichkeiten haben ( $N$ :) Durch längerfristige politische Arbeit sind die Gegensätze aufzuheben, d.h. durch Veränderung der Wirtschaftsordnung.“ (I. 0821) ,Die unterschiedliche Vermögensverteilung begründet Interessengegensätze. Eine Veränderung ist durch eine größere Beteiligung der Arbeitnehmer an dem Gewinn möglich. (N:) Es müßte über die bestehenden politischen Institutionen, über Gesetz, über gesetzliche Maßnahmen - auch Enteignung - verhindert werden, daß einige wenige Riesengewinne machen, wovon $90 \%$ der Bevölkerung nichts hat." (I. 0847) ,Interessengegensätze ergeben sich aus der übermächtigen Entwicklung von in sich verflochtenen Industrie- und Wirtschaftszweigen, gegenüber denen selbst der Staat ohnmächtig dasteht. (N:) Eine Veränderung ist durch staatliche Maßnahmen möglich: z.B. schrittweise Entflechtung und Verstaatlichung einzelner Bereiche, gerechtere Steuergebung, verstärkte Aufsicht gegenüber Monopolbetrieben." (I. 0875)

Die Ergebnisse zu den Deutungsmustern für gesellschaftliche Interessenunterschiede lassen sich in der folgenden Weise zusammenfassen. Auf der einen Seite überwiegen bei den Lehrern Erklärungsversuche, die sich vor allem auf Individuen beziehen und sozio-ökonomische Dimensionen nicht aufgreifen; drei Fünftel der Befragten entfallen auf die beiden ersten Deutungsmuster. Ausserdem zeigt sich ein relatives Übergewicht von Erklärungsversuchen, die von tendenziell harmonischen Ausgleichsvorstellungen ausgehen. Denn ebenfalls drei Fünftel der Antworten entfallen auf die beiden mittleren Kategorien. Dazu muß aber einschränkend angemerkt werden, daß in recht vielen der unter dem Deutungsmuster der sozio-ökonomischen Partnerschaft zusammengefaßten Aussagen der Konfliktgehalt von Interessenauseinandersetzungen angesprochen wird. Als dritter wichtiger empirischer Befund ist festzuhalten, daß nicht wenige Lehrer in ihren Erklärungsversuchen auf Naturkategorien zurückgreifen, was in der Besetzung des ersten Deutungsmusters zum Ausdruck kommt. Mit diesen Ergebnissen werden zum Teil Tendenzen bestätigt, die in älteren Untersuchungen zum Lehrer- bewußtsein herausgearbeitet wurden (E. Becker et al., 1967; M. Teschner, 1968; G. Schefer, 1967; A. Combe, 1971)

Auf der anderen Seite zeigen sich aber wichtige Elemente einer anderen Gesellschaftsvorstellung. Zwei Fünftel der Befragten beziehen sich auf sozio-ökonomische Grundverhältnisse der Gesellschaft und auf konkret zu bezeichnende gesellschaftliche Gruppen. Darüber hinaus werden von einem knappen Fünftel der Lehrer harmonische Ausgleichsvorstellungen mit dem Verweis auf soziale Ungleichheit problematisiert und außerdem politische Lösungsperspektiven entworfen. In diesen Befunden werden Tendenzen fortgesetzt, die sich in neueren Untersuchungen zum Lehrerbewußtsein gezeigt haben. (A. Hopf, 1974; B. Lehmann, 1974)

\section{Qualifikation, Selektion und gesellschaft- liche Wirklichkeit}

Die von den befragten Lehrern vorgenommene Interpretation und Auseinandersetzung mit den Aufgaben der Qualifikation und Auslese steht in einem Zusammenhang mit den übergeordneten gesellschaftlichen Deutungsmustern. Der objektiven Beziehung zwischen Schule und Gesellschaft, die durch die faktischen Prozesse der Sozialisation und Selektion hergestellt wird, entspricht eine subjektive Beziehung, die die Lehrer durch ihr pädagogisches Handeln und ihre pädagogischen Entwürfe eingehen. Dieser Zusammenhang wird zunächst für die Funktion der Selektion und dann für die Aufgabe der Qualifikation dargelegt (s. Tab. 8).

\section{Der Zusammenhang zwischen der Auseinander-} setzung mit der Auslesefunktion und den Deutungsmustern gesellschaftlicher Interessenunterschiede ist weniger prägnant ausgeprägt, als auf der Grundlage theoretischer Vorüberlegungen vermutet wurde. Durch eine genauere Betrachtung der einzelnen Beziehungen wird dieses empirische Ergebnis jedoch verständlich. Das gesellschaftliche Deutungsmuster, nach dem naturgegebene Unterschiede zwischen den Menschen die Grundlage von Interessenauseinandersetzungen abgeben, besitzt offensichtlich eine gleich starke Affinität zu dem problematischen und dem unproblematischen Verhältnis zur Auslese. Das deutet darauf hin, daß sich unter den Lehrern, die sich durch die Auslesefunktion in ihrer 
Tabelle 8: Auscinandersetzung mit der Selektionsfunktion und Deutungsmuster

\begin{tabular}{|c|c|c|c|}
\hline \multirow{2}{*}{$\begin{array}{l}\text { Deutungsmuster } \\
\text { gesellschaftlicher } \\
\text { Interessenunterschiede }\end{array}$} & \multicolumn{3}{|c|}{ Dilemma zwischen Förderung und Auslese } \\
\hline & Besteht & $\begin{array}{l}\text { Besteht nicht, } \\
\text { weil kein } \\
\text { Widerspruch }\end{array}$ & $\begin{array}{l}\text { Besteht nicht, } \\
\text { weil kein } \\
\text { Auslese }\end{array}$ \\
\hline $\begin{array}{l}\text { Individueller } \\
\text { Antagonismus }\end{array}$ & 16 & 17 & 17 \\
\hline Individueller Pluralismus & 37 & 45 & 34 \\
\hline $\begin{array}{l}\text { Sozio-ökonomische } \\
\text { Sozialpartnerschaft }\end{array}$ & 18 & 23 & 26 \\
\hline Sozio-ökonomische Ungleichheit & 24 & 9 & 17 \\
\hline Sonstiges & 6 & 5 & 6 \\
\hline $\begin{array}{r}\text { Summe } \\
\quad \mathrm{N}\end{array}$ & $\begin{array}{l}100 \\
497\end{array}$ & $\begin{array}{l}100 \\
438\end{array}$ & $\begin{array}{r}100 \\
47\end{array}$ \\
\hline
\end{tabular}

Arbeit belastet fühlen, einige Pädagogen befinden, die die negativen Folgen dieser Auslese als Ausdruck naturgegebener Unterschiede verstehen. Bei den drei anderen gesellschaftlichen Deutungsmustern läßt sich eine spezifische Beziehung zur Auseinandersetzung mit der Auslesefunktion erkennen. Die Problematisierung der Selektionsfunktion, die mit der Unterrichtstätigkeit verknüpft ist und mit der über die Verteilung der Bildung und Ausbildung auf die heranwachsende Generation entschieden wird, ist stärker als die unproblematische Interpretation mit einem Bewußtsein von sozialer Ungleichheit verbunden.

Bei der Beziehung zwischen gesellschaftlichem Deutungsmuster und Qualifikationsentwurf deutet sich ein Zusammenhang an, der schon in einem allgemeinen Vergleich erkennbar wird. Die genauere Bestimmung dieses Zusammenhangs geht von der schon angedeuteten theoretischen Beziehung aus. Auf der einen Seite impliziert die mit den Deutungsmustern vorgestellte so-

Tabelle 9: Qualifikationsziele und gesellschaf̣tliche Deutungsmuster

\begin{tabular}{llllll}
\hline $\begin{array}{l}\text { Qualifikation für } \\
\text { Arbeit und Beruf }\end{array}$ & $\begin{array}{l}\text { Deutungsmuster gesellschaftlicher Interessenunterschiede } \\
\text { Individueller } \\
\text { Antagonismus }\end{array}$ & $\begin{array}{l}\text { Individueller } \\
\text { Pluralismus }\end{array}$ & $\begin{array}{l}\text { Sozio-ökonom. } \\
\text { Sozialpartner- } \\
\text { schaft }\end{array}$ & $\begin{array}{l}\text { Sozio-ökonom. Sonstiges } \\
\text { Ungleichheit }\end{array}$ & $\begin{array}{l}\text { Summe } \\
\%\end{array}$ \\
\hline
\end{tabular}

Arbeitstugenden und instrumentelle Qualifikationen

27

23

Qualifikationen

Selbständige Anwendung der instrumentellen Qualifikationen

Selbständig-kooperative Anwendung der instrumentellen Qualifikation

Arbeitsqualifikation und Fähigkeit zur Durchsetzung von Arbeitnehmerinteressen 
ziale Wirklichkeit je spezifische Verhaltens- und Handlungsweisen der in ihr lebenden Gesellschaftsmitglieder. Auf der anderen Seite werden mit Sozialisationszielen die auszubildenden Verhaltens- und Handlungsfähigkeiten formuliert und damit deren Notwendigkeit für die gesellschaftliche Existenz postuliert. Sozialisationsentwurf heißt also immer auch Gesellschaftsentwurf. (M.R. Vogel, 1970)

Der empirische Zusammenháng zwischen Qualifikationsentwurf und gesellschaftlichem Deutungsmuster (s. Tab. 9) wird besonders deutlich bei den beiden entgegengesetzten Vorstellungen einer im Unterricht zu leistenden Vorbereitung auf Arbeit und Beruf. Er ist aber auch bei den dazwischenliegenden Qualifikationsentwürfen erkennbar. Die überwiegende Mehrheit der Lehrer, die die Arbeitstugenden hervorhebt, verwendet individuelle Deutungsmuster für die gesellschaftlichen Interessenunterschiede. Außerdem findet bei diesen Lehrern der Verweis auf naturgegebene Gegensätze die größte Verbreitung. Der von diesen Lehrern vertretene Qualifikationsbegriff, mit dem die Notwendigkeit zur Einpassung und Unterordnung betont wird, scheint diesen Gesellschaftsvorstellungen am ehesten zu entsprechen. Er läßt sich wesentlich seltener mit Gesellschaftsvorstellungen verbinden, nach denen Interessen auf gesellschaftliche Gruppen und auf sozio-ökonomische Grundlagen zu beziehen sind. Denn zu einer solchen Vorstellung von gesellschaftlichen Auseinandersetzungen gehören Menschen mit Fähigkeiten zur Wahrnehmung eigener und fremder Interessen und mit Fähigkeiten zur Gestaltung sozialer Wirklichkeit.

Der Übergang zu den Vertretern der drei mittleren Qualifikationsentwuirfen ist mit einer $\mathrm{Zu}$ nahme sozio-ökonomischer Deutungsmuster verbunden. Die Unterschiede zwischen diesen drei Zielsetzungen sind mit der Tendenz zur Abnahme desjenigen Erklärungsversuchs verknüpft, mit dem vor allem naturgegebene Unterschiede hervorgehoben werden. Die Interpretation gesellschaftlicher Wirklichkeit, nach der Interessenkämpfe mit naturgegebenen Gegensätzen zwischen den Menschen begründet werden, wird mit einem Qualifikationsbegriff unvereinbar, in dem die Eingrenzung auf instrumentelle Aspekte überwunden ist. Das zeigt sich bei dem Aufgreifen der Selbständigkeit und bei der Berücksichtigung der Kooperationsfähigkeit. Die mit dieser Veränderung des Qualifikationsbegriffs verbundede Ausweitung der arbeitenden Person gerät in Widerspruch zu einer tendenziell ahistorischen Vorstellung von gesellschaftlichen Interessenauseinandersetzungen.

Der entscheidende Übergang von den individuellen zu den sozio-ökonomischen Deutungsmustern gesellschaftlicher Interessen vollzieht sich bei denjenigen Lehrern, die in ihre Zielsetzung neben der Arbeitsfähigkeit auch die Fähigkeit zum interessengeleiteten Handeln aufnehmen. Die überwiegende Mehrheit der Lehrer, die die Schüler in diesem Sinne auf ihre zukünftige Arbeitsexistenz vorbereiten wollen, haben eine $\mathrm{Ge}$ sellschaftsvorstellung, nach der gesellschaftliche Interessen auf soziale Gruppen und auf eine ökonomische Basis zu beziehen sind. Zwischen den beiden sozio-ökonomischen Deutungsmustern zeigt sich ein leichtes Übergewicht bei derjenigen Variante, mit der die soziale Ungleichheit hervorgehoben wird. Dieses zweite sozio-ökonomische Deutungsmuster muß sich besonders eng mit Sozialisationsvorstellungen verbinden, in denen Aspekte des Interessenhandelns aufgegriffen werden. Denn mit diesem Deutungsmuster wird Gesellschaft als eine historisch-soziale Wirklichkeit vorgestellt, die von den Gesellschaftsmitgliedern mit den Mitteln der Politik zu gestalten ist. Der zusammenfassende Überblick macht deutlich, daß mit dem Durchlaufen der verschiedenen Qualifikationsentwürfe eine genaue Umkehrung der Gesellschaftsvorstellungen verbunden ist.

Mit den hier vorgelegten empirischen Ergebnissen konnte gezeigt werden, wie sich die Lehrer mit zwei zentralen Funktionen ihrer Unterrichtstätigkeit auseinandersetzen. Diese Ergebnisse lassen sich einerseits vergleichen mit Ergebnissen, die sich aus den Ansätzen zu einer objektiven Funktionsbestimmung der Schule ergeben. Sie lassen sich andererseits mit pädagogischen und politischen Vorstellungen von einer sinnvollen schulischen Sozialisation der heranwachsenden Generation konfrontieren. Außerdem konnte gezeigt werden, daß die Auseinandersetzung der Lehrer mit den gesellschaftlichen Funktionen des Unterrichts über allgemeine schulische Rahmenbedingungen hinaus von subjektiven und objektiven Bedingungen abhängig ist, die sich aus Fach und Schulart ergeben. Schließlich konnte deutlich gemacht werden, wie die Auslegung der gesellschaftlichen Funktionen des 
Unterrichts mit Vorstellungen von der Gesellschaft zusammenhängen. So ergibt sich aus der Verknüpfung von Arbeitsbewußtsein und gesellschaftlichen Deutungsmustern ein wichtiger Zugang sowohl zum pädagogischen als auch zum gesellschaftlichen Bewußtsein der Lehrer.

\section{Literatur}

Combe, A., 1971: Kritik des Lehrerbewußtseins, Gesellschaftliche Voraussetzungen und soziale Folgen des Lehrerbewußtseins. München.

Becker, E., Herkommer, S., Bergmann, J., 1967: Erzichung zur Anpassung? Eine soziologische Untersuchung der politischen Bildung in den Schulen. Frankfurt/M.

Engelhardt, M. v., 1978: Die Unterrichtsarbeit des des Lehrers und das Leistungsprinzip. In:J. Beck/ H. Boehncke (Hersg.) : Jahrbuch für Lehrer 1979. Reinbeck

Engelhardt, M. v., 1979: Lernziel: Mündige Schüler. betrifft: erziehung 2, 50-54
Engelhardt, M. v. und R.-W. Hoffmann, 1974: Wissenschaftlich-technische Intelligenz im Forschungsgroßbetrieb. Frankfurt/M..

Fend, H., 1974: Gesellschaftliche Bedingungen schulischer Sozialisation. Weinheim und Basel.

Hopf, A., 1974: Lehrerbewußtsein im Wandel. Düsseldorf.

Kob, J.-P., 1958: Das soziale Bewußtsein der Lehrer an Höheren Schulen. Würzburg.

Koch, J.J., 1973: Lehrerstudium und Beruf. Einstellungswandel in den ersten beiden Phasen der Ausbildung. Ulm.

Kratzsch, E.H., Vathke, W. und Bertlein, H., 1967: Studien zur Soziologie des Volksschullehrers. Weinheim.

Lehmann, B., 1974: Arbeitswelt und Lehrerbewußtsein. Neuwied und Berlin.

Schefer, B., 1974: Das Gesellschaftsbild des Gymnasiallehrers. Frankfurt/M..

Teschner, M., 1968: Politik und Gesellschaft im Unterricht. Eine soziologische Analyse der politischen Bildung an hessischen Gymnasien. Frankfurt/M..

Vogel, M.R., 1970: Erziehung im Gesellschaftssystem. München.

Vonderach, G., 1972: Industriearbeit und Lehrerausbildung. Eine empirische Untersuchung zur politischen Bewußtseinsbildung künftiger Lehrer. Stuttgart. 